\title{
Avaliação de conhecimentos e hábitos de proteção solar de crianças e adolescentes
}

Cátia Alexandra Granja Ferreira, ${ }^{1}$ Filipa Inês de Sousa Vela Cunha ${ }^{2}$

\section{RESUMO}

Objetivo: Avaliar e descrever os conhecimentos e hábitos de proteção solar em idade pediátrica.

Tipo de estudo: Estudo observacional transversal analítico.

Local: Consulta externa e serviço de urgência de pediatria de um hospital nível Il e consulta de saúde infantil de uma Unidade de Saúde Familiar (USF) local.

População: Crianças com idade inferior a 18 anos e respetivos acompanhantes que frequentaram as unidades referidas.

Métodos: Aplicação de um questionário sobre conhecimentos e hábitos sobre proteção solar de resposta voluntária e confidencial. O tratamento estatístico dos dados foi realizado através do programa Statistical Package for the Social Sciences - SPSS ${ }^{\circledR}$. Considerou-se existir significância estatística para valores de $p<0,05$.

Resultados: Amostra de 295 questionários, 63,7\% respondidos por cuidadores de crianças com idade inferior a nove anos e $36,3 \%$ por adolescentes entre os 10 e os 17 anos. O período do dia de maior exposição solar foi antes das 11 horas e após as 16 horas (77\%). Usam protetor solar 93\% dos adolescentes e 90,6\% das crianças; no entanto, a maioria da população usa-o apenas na praia $(61 \%)(p<0,05)$. Quanto à frequência de renovação de protetor solar, $40,2 \%$ dos adolescentes renovam duas vezes e $52,6 \%$ das crianças apenas uma vez $(p<0,05)$. É o sexo feminino que usa mais o guarda-sol $(76,2 \%)(p<0,05)$ e que utiliza fator de proteção solar (FPS) 30 (59\%) e 50+ (73,7\%), enquanto mais rapazes utilizam o FPS $<15(76,9 \%)(p<0,05)$. Os questionados referiram obter mais informação sobre proteção solar através da comunicação social $(46,8 \%)$.

Discussão/Conclusão: Este estudo demonstrou hábitos inadequados de proteção solar, resultados concordantes com outros estudos. Alerta-se para a necessidade de divulgação mais ampla de conhecimentos adequados que consolide práticas saudáveis em relação à exposição solar. A multidisciplinaridade entre os cuidados de saúde primários e a pediatria torna-se fulcral.

Palavras-chave: Crianças; Conhecimento; Hábitos; Luz solar; Agentes de proteção solar.

\section{INTRODUÇÃO}

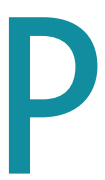

assar tempo ao ar livre é uma parte essencial de um estilo de vida ativo e saudável para todas as faixas etárias. É também a melhor forma de manter níveis adequados de vitamina D. ${ }^{1}$ No entanto, a exposição inadequada ao sol pode levar a queimaduras solares e a exposição ultravioleta (UV) a ter outros efeitos deléterios para a saúde, particularmente pela sua ação carcinogénica, aumentando o risco de melanoma maligno, tumores queratinocíticos e carcinomas basocelular e espinocelular. ${ }^{1}$

1. Médica Interna de Pediatria. Serviço de Pediatria, Hospital Distrital da Figueira da Foz. Figueira da Foz, Portugal.

2. Assistente de Pediatria. Serviço de Pediatria, Hospital Distrital da Figueira da Foz. Figueira da Foz, Portugal.
Desde a década de 1920 as tendências de estilo de vida e a moda entre as populações de pele clara têm impulsionado a exposição direta e deliberada de grandes períodos ao sol, especialmente em adolescentes e adultos jovens. Viagens mais baratas para destinos de praia, estilos de roupa com mais pele exposta e a busca por um bronzeado na moda, através da exposição solar deliberada, contribuíram significativamente para aumentar as taxas de cancro de pele em adultos de pele clara ao redor do mundo. ${ }^{1-2}$

Embora a maioria dos cancros de pele seja detetada nos adultos também podem surgir em crianças e adolescentes. Anualmente são diagnosticados cerca de dois a três milhões de casos de cancro cutâneo não-melanoma e 130.000 casos de melanoma. ${ }^{2}$ Segundo os 
dados da Globocan (2018), em Portugal foram diagnosticados 1.329 (356 fatais) melanomas cutâneos, correspondendo a uma taxa de incidência (padronizada para a idade) de 6,7 no mesmo ano. ${ }^{3}$ Em Portugal estima-se que a incidência do melanoma seja de 10 novos casos por 100.000 habitantes por ano, o que significa cerca de 1.000 novos casos por ano. Contudo, há problemas na notificação dos casos de melanoma, uma vez que nem todos os doentes são tratados a nível hospitalar devido a crescente procura de cuidados de saúde pela população, sendo que hoje em dia uma percentagem importante de consultas, exames e tratamentos é realizada em instituições privadas e por vezes não notificada. Em relação aos carcinomas, basocelular e espinocelular, estima-se uma prevalência de mais de 100 novos casos por 100.000 habitantes, o que representa mais de 10.000 novos casos por ano. Os dados são calculados usando taxas específicas por idade e correspondentes populações por 10 grupos etários. Assim, o cancro de pele em geral representa o cancro humano mais frequente..$^{3-4} \mathrm{O}$ cancro cutâneo não-melanoma relaciona-se diretamente com a acumulação de exposição UV ao longo da vida, enquanto o risco de melanoma maligno e, por outro lado, mais elevado com exposições intermitentes e intensas, sobretudo antes dos 20 anos de idade. ${ }^{6-7} \mathrm{~A}$ exposição aos raios UV desde o início de vida tem efeitos deletérios cumulativos na pele, mas também pode resultar em catarata, sendo importante proteger os olhos jovens da exposição à radiação. ${ }^{8}$ As queimaduras solares e as práticas inadequadas de fotoproteção são comuns, especialmente entre os adolescentes, em diferentes séries internacionais. ${ }^{9}$

Portugal tem um clima temperado mediterrânico, influenciado pela latitude, relevo e pela proximidade ao Oceano Atlântico. A precipitação e a temperatura variam ao longo das regiões, de norte a sul e de oeste a este, apresentando uma grande variabilidade sazonal e anual. A temperatura média desde os anos 70 tem vindo a subir em todas as regiões de Portugal a uma taxa de cerca de $0,3^{\circ} \mathrm{C}$ por década. Verifica-se o aumento de número de dias com temperaturas altas e redução de números de dias com temperaturas baixas, facto que está relacionado com o aumento na intensidade e duração das ondas de calor. ${ }^{10}$

Os pais têm a responsabilidade de garantir a proteção solar dos seus filhos durante os primeiros anos de vida. Eles também são modelos importantes para comportamentos relacionados com a saúde, em particular ao praticarem bons hábitos em relação à proteção contra a radiação UV, seja através do uso de meios físicos (roupa adequada, chapéus, óculos de sol...) ou do protetor solar. A literatura considera os hábitos parentais o fator essencial para a fotoproteção das criancas. ${ }^{11-12}$

Assim, os objetivos deste trabalho foram avaliar os conhecimentos e hábitos dos cuidadores e das próprias crianças/adolescentes, relativamente à proteção solar e procurar estabelecer uma relação entre esses conhecimentos e diferentes variáveis [género, idade, escolaridade, profissão, fototipos de Fitzpatrick, presença de efélides, lesões na pele, horário de exposição ao sol, uso de protetor solar e fator de protetor solar (FPS), uso de outros meios físicos de proteção solar], bem como investigar a maior fonte de informação sobre proteção solar.

\section{MÉTODOS}

Foi realizado um estudo analítico transversal visando conhecer os hábitos da população pediátrica e seus cuidadores, face aos cuidados relacionados com a proteção solar.

A população de estudo foi composta por indivíduos com idade inferior a 18 anos e respetivos acompanhantes que frequentaram as consultas externas e o serviço de urgência de pediatria do Hospital Distrital da Figueira da Foz e as consultas de saúde infantil e juvenil da Unidade de Saúde Familiar de Buarcos - Figueira da Foz no período de tempo compreendido entre 1 de agosto e 30 de setembro de 2018.

Procedeu-se à aplicação de um questionário escrito anónimo, de preenchimento voluntário e aprovado pela comissão de ética do Hospital Distrital da Figueira da Foz. Tratou-se de um questionário adaptado e construído para o efeito, tendo como base o The Sun Exposure and Protection Index (SEPI). ${ }^{13}$

Para a análise dos dados foi utilizada a versao 23.0 do programa estatistico SPSS (Statistical Package for the Social Science, Chicago, IL, EUA). Inicialmente foram aplicados conceitos de estatística descritiva, recorrendo a frequências absolutas ( $n$ ) e relativas (\%) para as variáveis qualitativas e medidas de tendência central para as variáveis quantitativas. Posteriormente aplicaram-se técnicas de inferência estatística com o objetivo de 


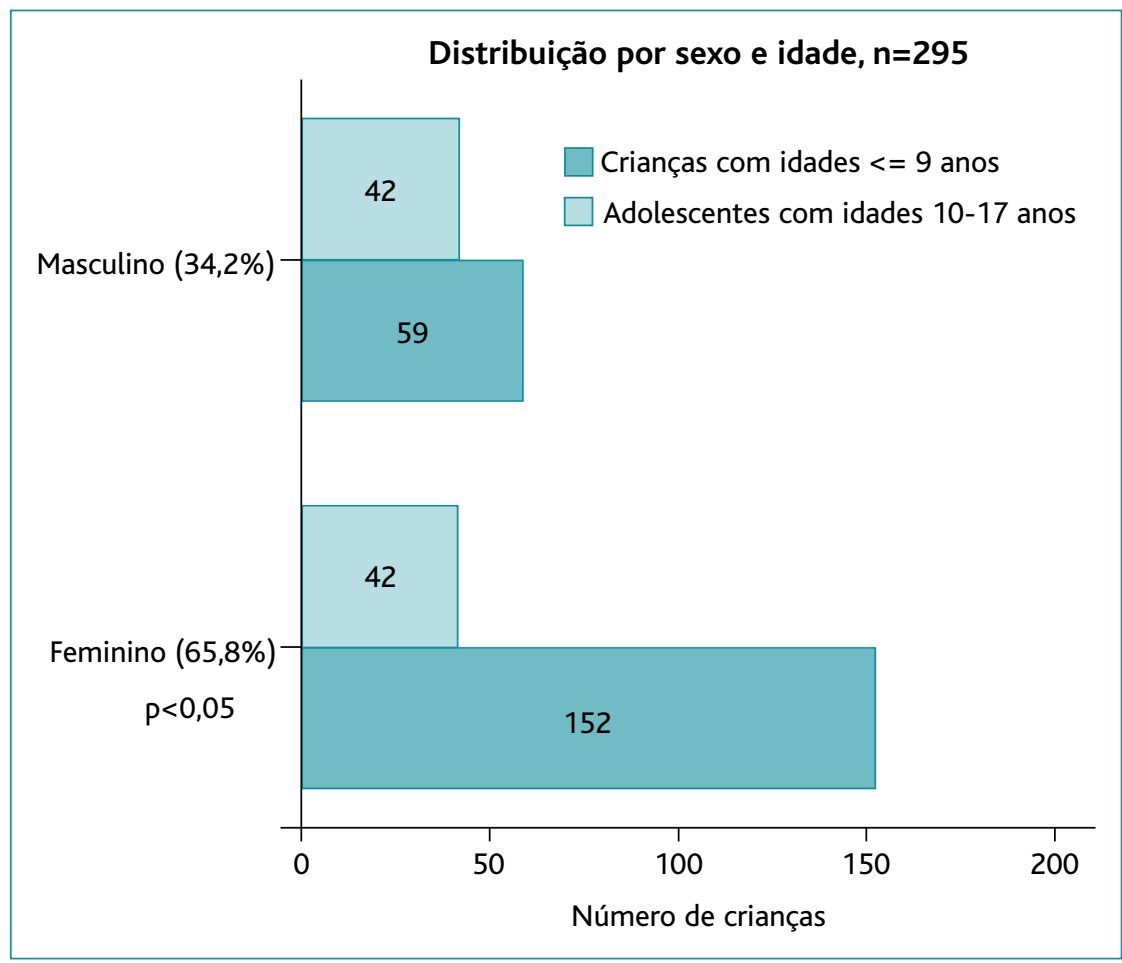

Figura 1. Distribuição do total da amostra $(n=295)$ por sexo e idade. de crianças com idade inferior a nove anos foi o $12^{\circ}$ ano $(42,4 \%) \mathrm{e}$ $37,9 \%$ possuíam licenciatura. Pôde constatar-se que o setor económico profissional do cuidador predominante foi o setor terciário $(59,1 \%)$ e $9 \%$ dos inquiridos encontravam-se desempregados.

Quando questionada a presença de algum problema de pele, apenas $21,4 \%$ do total da amostra respondeu afirmativamente e $14,2 \%$ tinha efélides.

Os fotótipos de pele mais frequentes foram o fototipo III $(45,8 \%)$ e o IV $(27,8 \%)$ (Figura 2$)$.

\section{Comportamento}

Verificou-se que $77 \%$ dos inquiridos têm um bom conhecimento acerca da melhor hora para ir para a praia, identificando a resposta correta (antes das $11 \mathrm{~h}$ e/ou depois das $16 \mathrm{~h}$ ) de entre as várias opções

validar as hipóteses em investigação. Para a análise de variáveis qualitativas usou-se o teste do Qui-quadrado e para variáveis quantitativas usou-se o teste $t$-student para amostras independentes. A tomada de decisão foi feita com base no valor do $p$-value, considerando como nível de significância $p<0,05$.

\section{RESULTADOS}

\section{Participantes}

Neste estudo foi utilizada uma amostra de conveniência com 295 questionários validados.

A amostra foi dividida em dois grupos de idades: cuidadores de crianças com idades inferiores a nove anos $(n=188,63,7 \%)$ e adolescentes com idades compreendidas entre os 10 e os 17 anos $(n=107,36,3 \%$, média de idades de $14 \pm 2$ anos - mínimo de 10 e máximo de 17), com um predomínio do sexo feminino $(65,8 \%)$ (Figura 1). O grau de escolaridade mais frequente dos cuidadores de resposta.

O estudo revelou que a maioria dos inquiridos usa protetor solar, $93 \%$ dos adolescentes e $90,6 \%$ das crianças com idade inferior a nove anos; no entanto, a

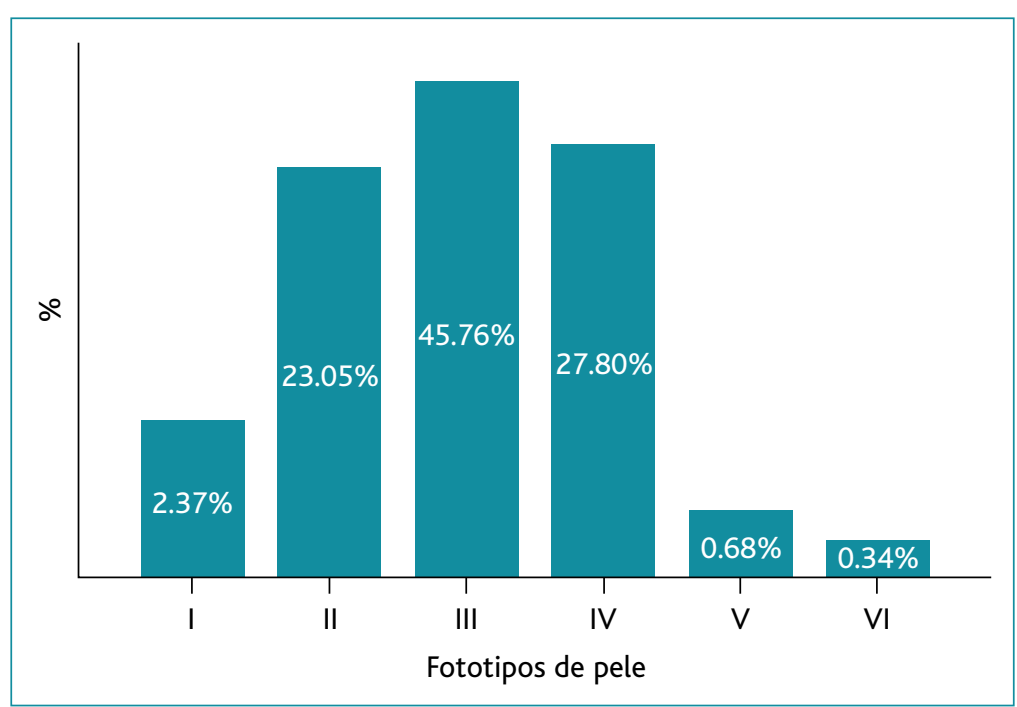

Figura 2. Fototipos de pele mais frequentes, $n=295$. 


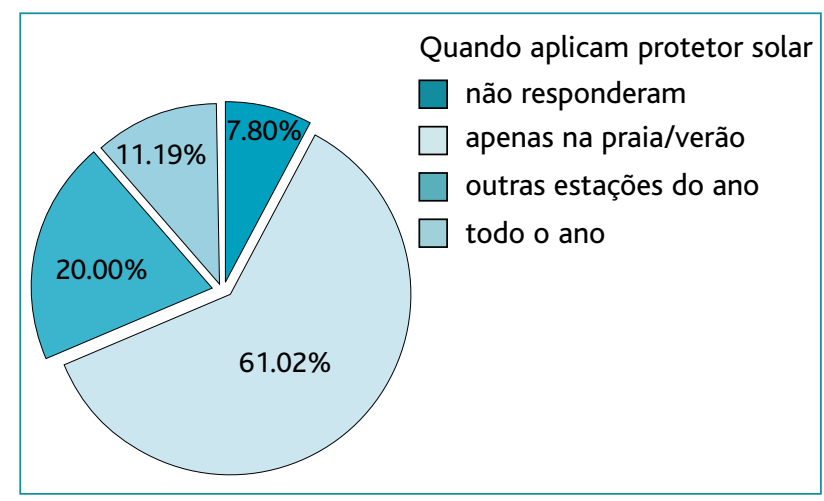

Figura 3. Momento temporal de aplicação de protetor solar ao longo do ano, $n=295(p<0,05)$.

maioria da população usa-o apenas na praia (61\%) $(p<0,05)$. Dos adolescentes, $64 \%$ usam protetor solar apenas na praia/Verão e $4,1 \%$ todo o ano (Figura 3). Quanto à frequência de renovação de protetor solar, $40,2 \%$ dos adolescentes renovam duas vezes e $52,6 \%$ das crianças apenas uma vez $(p<0,05)$ (Figura 4$)$.

Acerca do FPS, $4,4 \%$ usam FPS $\leq 15,20,7 \%$ usam FPS $30+$ e $67,1 \%$ usam FPS $\geq 50$, havendo correlação entre fototipos de pele mais sensíveis (I, II, III) e FPS mais elevados $(\geq 50)(p<0,05)$. Interrogados acerca do tipo de pele mais propício ao eritema actínico, $1,5 \%$ da amostra apontou ser a pele negra, $6,2 \%$ indicou a pele morena, $49,3 \%$ considerou a pele clara e $43,1 \%$ apontou a pele muito clara.

A maioria dos sujeitos considerou que usar chapéu e guarda-sol é importante. De facto, $61,7 \%$ dos inquiridos indicaram o chapéu como essencial num dia de praia e $60,7 \%$ o guarda-sol. Em contraste, $72,5 \%$ dos inquiridos não consideraram importante o uso de $(p<0,05)$. camisola e 54,9\% negam o uso de óculos de sol (Figura 5).

Quando questionados relativamente ao local onde obtiveram maior informação sobre proteção solar, $46,8 \%$ dos inquiridos referiram obter maior informação através da comunicação social, sendo a maioria cuidadores de crianças com idade inferior a nove anos $(p<0,05), 38 \%$ mencionam conhecimento próprio e $20,1 \%$ através de aconselhamento em farmácia. Apenas $17,3 \%$ responderam o pediatra, $14,6 \%$ referem o médico/enfermeira de família e $6,4 \%$ responderam outro meio de informação, sendo estes últimos jovens que mencionam a escola como fonte de conhecimento sobre proteção solar.

\section{Sexo/Comportamento}

Os resultados revelam a existência de uma relação entre o uso de protetor solar e o sexo, mostrando que $60,9 \%$ dos inquiridos que não usam protetor solar são do sexo masculino $(p<0,05)$. Adicionalmente, uma relação entre o sexo e o FPS do protetor foi também encontrada $(p<0,001)$, sendo que o sexo feminino utiliza mais FPS 50+ $(73,7 \%)$, enquanto o sexo masculino utiliza mais o FPS $<15(76,9 \%)$.

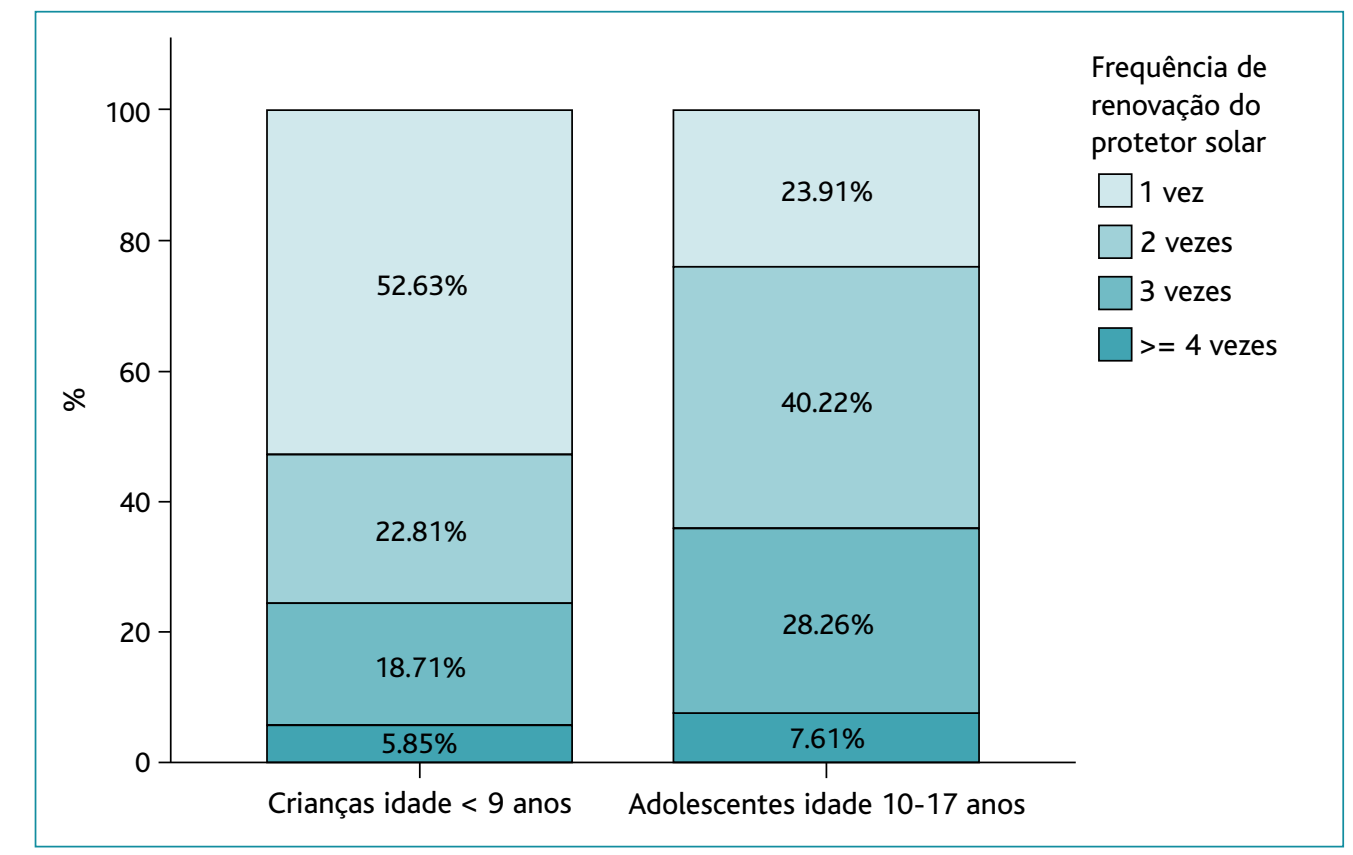

Figura 4. Frequência de renovação do protetor solar, durante um dia de exposição solar, por idades, $n=295$ 


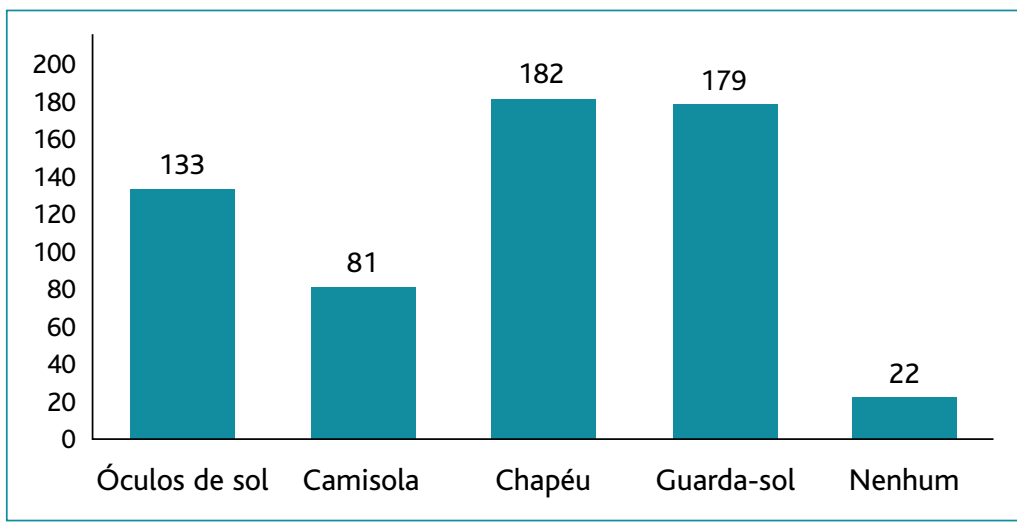

Figura 5. Uso de outros meios físicos de fotoproteção, para além do protetor solar.

trando, assim, a pertinência da proteção solar nesta faixa etária. A investigação mostra um conhecimento significativo da população acerca do tema, o que, no entanto, não se reflete em práticas e medidas de proteção adequadas, nomeadamente no uso de roupa adequada, chapéu, óculos de sol ou na frequência de renovação do protetor solar na exposição prolongada ao sol. ${ }^{14}$

Da análise dos 295 questionários, a maioria cuidadores de crianças com idade inferior a nove anos de idade, do sexo feminino e com tipo de pele predominante

Não se verificou uma associação entre o sexo e o número de vezes que renovam o protetor solar num dia de praia.

Foi ainda constatada uma relação entre o sexo e o conhecimento sobre a importância de usar guarda-sol $(p<0,001)$. Assim, é o sexo feminino que usa mais o guarda-sol $(76,2 \%)$, não se verificando associação entre o sexo e o uso dos restantes meios de fotoproteção (óculos, camisola ou chapéu).

\section{Faixa etária/Comportamento}

Não se verificou associação estatisticamente significativa entre a faixa etária e o uso de protetor solar ou o FPS mais utilizado; no entanto, dentro do grupo dos inquiridos que não usa protetor solar $(n=23) 65,2 \%$ são adolescentes.

Relativamente à renovação de protetor solar num dia de praia, encontrou-se uma relação com a idade $(p<0,001)$. Esta proteção é renovada mais vezes pelos adolescentes.

Verificou-se ainda uma associação entre a idade e o uso de guarda-sol $(p<0,001)$. Desta forma, dos que usam guarda-sol, 78,6\% são cuidadores de crianças com idade inferior a nove anos e, dos que não usam guarda-sol, $57,1 \%$ têm idade compreendida entre 13 e 17 anos.

Não se verificou associação entre a faixa etária e uso dos restantes meios de fotoproteção (óculos, camisola ou chapéu).

\section{DISCUSSÃO}

Sabe-se que na infância e adolescência a exposição à radiação UV é maior do que em outras idades, mos- branca/morena clara, fototipo III e IV, concluem-se alguns pontos positivos e outros menos positivos.

\section{Pontos positivos}

A maioria da população estudada parece ter bons conhecimentos acerca da importância da proteção solar. No que diz respeito ao protetor solar usado, a maioria dos inquiridos usa protetor solar, $93 \%$ dos adolescentes e 90,6\% das crianças com idade inferior a nove anos e os FPS mais usados correspondem aos FPS $30 \mathrm{e}$ $50+$, em concordância com a literatura e atuais recomendações. ${ }^{15}$

No presente estudo, fototipos de pele mais clara (I, II, III) usam FPS mais elevado $(>30)(p<0,05)$, como em estudos semelhantes; mesmo em pessoas de diferentes idades o uso de filtro solar esteve associado à cor da pele. ${ }^{16-17}$

Relativamente ao horário e tempo de exposição solar, no presente estudo a maioria refere exposição solar em horário adequado $(77 \%$ antes das $11 \mathrm{~h}$ e/ou após as 16h), ainda que se verifique que uma percentagem considerável (23\%) tem exposição entre as 11 e as 16 horas. Assim, comparativamente com outros estudos, a presente população em análise parece mais consciencializada sobre os horários adequados de exposição. Dois estudos mostram, respetivamente, $37,3 \%$ das crianças com exposição solar entre as 10 e as 16 horas e outro em que o período de maior exposição foi precisamente entre as 10 e 16 horas $(65,5 \%) .{ }^{15,18}$

\section{Pontos menos positivos}

Não foi encontrada associação estatística entre o tipo 
de pele e a frequência de renovação do protetor solar ou o seu uso durante todo o ano. O nível de escolaridade não se relacionou com a frequência de renovação.

Verifica-se também que a maior parte das crianças em estudo não usa outras medidas de proteção solar adicionais, para além do protetor solar. Apenas o guarda-sol foi mencionado maioritariamente pelo sexo feminino e principalmente por adultos, assim como no estudo de Silva e colaboradores onde verificaram que $66,7 \%$ das crianças não fazem uso de medidas de proteção adicional. ${ }^{18}$ Tresoldi, Duarte e Costa observaram que as mulheres possuem um maior conhecimento sobre os danos da radiação UV e se preocupam mais em proteger-se que os homens. ${ }^{19}$ Estes resultados fazem pensar que os programas de prevenção, as campanhas de sensibilização ou mesmo a publicidade acerca do uso do protetor sol pode estar a reforçar a crença de que o uso de protetor solar por si só é suficiente para proteger a pele da radiação UV e prevenir o cancro cutâneo, o que não é verdade.

Uma percentagem muito pequena de crianças faz uso do protetor solar diariamente, parecendo usá-lo apenas quando há exposição direta ao sol. O uso de protetor solar é pouco utilizado pelos adolescentes, sendo que $64 \%$ admite que usa protetor solar apenas na praia e apenas $4,1 \%$ o usa durante todo o ano $(p<0,05)$. Um estudo realizado em Portugal com 177 adolescentes mostrou que $44,2 \%$ diz nunca ou raramente usar protetor solar, sendo que somente $13,5 \%$ afirma usar frequentemente protetor solar (quase todos os dias ou todos os dias). ${ }^{20}$

Importa ainda destacar o papel dos meios de comunicação social na informação da população, citada por 46,8\% dos inquiridos, o que está em consonância com outros estudos nos quais a percentagem encontrada foi ainda mais elevada. A comunicação social continua a ser referida como o principal meio de educação para a saúde nesta área, seguido do conhecimento próprio (38\%), farmácia $(20,1 \%)$, pediatra $(17,3 \%)$, médico de família/enfermeiro de família $(14,6 \%)$ e escola $(6,4 \%)$. Este valor encontra-se muito aquém do que seria o ideal ou, pelo menos, expectável, uma vez que o médico de família e o pediatra têm um papel primordial na prevenção.

Alguns estudos realizados por não dermatologistas sugerem que o uso de filtro solar para a prevenção do cancro de pele poderia expôr as pessoas ao risco de desenvolver deficiência de vitamina $\mathrm{D}$, sujeitando os pacientes a alterações na mineralização óssea. ${ }^{21-22}$ A esse respeito, um estudo realizado no Brasil (2007) indicou que práticas de proteção solar estiveram associadas a concentrações menores de 25-hidroxivitamina D (25OHD) em comparação com indivíduos expostos a luz solar, mas não o suficiente para causar deficiência de $250 H D .{ }^{23}$ Desta forma, atitudes que desencorajem o uso de filtro solar e barreiras físicas de proteção solar não devem ser recomendadas ou encorajadas.

Cerca de $50-80 \%$ das alterações induzidas pela exposição solar que um indivíduo recebe ao longo de toda a vida ocorrem durante a infância e a adolescência. É fundamental uma intervenção precoce relativamente à fotoproteção, que inclui todas as medidas que nos permitem proteger das radiações solares, quer através de meios físicos/barreira quer através do uso correto de protetor solar. Com dados semelhantes, outros estudos consideram os hábitos parentais o fator mais determinante para a fotoproteção das crianças. ${ }^{12,24}$

Sugere-se a implementação de programas que permitam intervir no seio familiar e na comunidade educativa, de modo a criar uma consciencialização sobre os efeitos da radiação UV e o incentivo de mudança de estilos de vida. Estes programas vêm ao encontro dos desejos expressos pela Organização Mundial da Saúde, referenciados na literatura, segundo os quais "as práticas saudáveis sobre a proteção solar adquiridas na escola ganham mais consistência se houver informação e sustentação por parte da família e da comunidade". ${ }^{25}$ Outro estudo realça ainda o impacto do suporte familiar no controlo e prevenção do cancro de pele, no qual segundo o artigo é significativo e deve ser considerado na criação de campanhas de sensibilização, em especial em termos de tentar envolver toda a família na difusão de métodos de prevenção. ${ }^{26}$

A proteção solar é um tema inquietante a nível mundial, sendo na maior parte das vezes relacionado com a praia e o lazer, esquecendo a atividade profissional. As profissões ao ar livre, agricultores, polícias, pescadores, construtores civis, vendedores de rua, carteiros, requerem cuidados especiais de proteção solar durante todo o ano. Um incentivo ao uso de protetor solar seria, por exemplo, a redução do IVA dos mesmos para $6 \%$. O estudo de Silva e colaboradores (de 2018) supõe que 
as pessoas não reaplicam o protetor solar pelos altos valores dos produtos no mercado. ${ }^{27}$

Este estudo apresenta limitações que é necessário considerar. $\mathrm{O}$ reduzido tamanho da amostra e a utilização de medidas de autorrelato para a avaliação da proteção solar, por exemplo, podem ser enviesadas por fatores como a honestidade na recordação de informação e a desejabilidade social. Importa também salientar que a aplicação do inquérito ocorreu durante os meses de agosto e setembro, na cidade da Figueira da Foz, em plena época balnear, uma altura de maior preocupação relativamente à exposição solar, ainda que, tendo em conta o clima português, a exposição solar esteja presente praticamente em todos os meses do ano.

\section{CONCLUSÃO}

Em conclusão, este estudo permitiu demonstrar que ainda existe um longo caminho a percorrer acerca desta temática. A dificuldade de transformar conhecimentos em práticas saudáveis é um desafio que ultrapassa as barreiras nacionais e culturais. A mudança de hábitos é, sem dúvida, um processo lento, mas que somente será possível com a participação ativa da sociedade, em que pese a responsabilidade dos médicos, independentemente da sua área de atuação, técnicos de saúde, comunicação social, farmácias e comunidade escolar de forma a promover bons hábitos de proteção solar em Portugal.

\section{REFERÊNCIAS BIBLIOGRÁFICAS}

1. World Health Organization, World Meteorological Organization, United Nations Environment Programme, International Commission on Non-Ionizing Radiation Protection. Global solar UV index: a practical guide [Internet]. Geneva:WHO; 2002. Available from: https://apps.who. int/iris/handle/10665/42459

2. World Health Organization. INTERSUN - The global UV project: a guide and compendium [Internet]. Geneva: World Health Organization; 2003. Available from: https://apps.who.int/iris/handle/10665/42814

3. Ferlay J, Soerjomataram I, Ervik M, Dikshit R, Eser S, Mathers C, et al. GLOBOCAN 2018: cancer incidence and mortality worldwide (IARC CancerBase no. 11) [homepage]. Lyon: International Agency for Research on Cancer; 2018. Available from: http://globocan.iarc.fr

4. Correia O.Agressao solar da pele por Osvaldo Correia [homepage]. Porto: Associacao Portuguesa de Cancro Cutaneo; 2016. Available from: http://www.apcancrocutaneo.pt/index.php/prevencao/saiba-mais/28agressao-solar-da-\%20pele-por-osvaldo-correia

5. Moura C. Melanoma por Cecilia Moura [homepage]. Porto:Associacao Portuguesa de Cancro Cutaneo; 2016. Available from: http://www.ap- cancrocutaneo.pt/index.php/saiba-mais/artigos-de-opiniao/29-melanoma-por-cecilia-moura

6. Committee on Environmental Health, American Academy of Pediatrics. Ultraviolet light: a hazard to children. Pediatrics. 1999;104(2 Pt 1):328-33.

7. Cravo M, Moreno A, Tellechea O, Cordeiro MR, Figueiredo A. Fotoprotecção na criança [Photoprotection in children]. Acta Pediatr Port. 2008;39(4):158-62. Portuguese

8. Linetsky M, Raghavan CT, Johar K, Fan X, Monnier VM, Vasavada AR, et al. UVA light-excited kynurenines oxidize ascorbate and modify lens proteins through the formation of advanced glycation end products: implications for human lens aging and cataract formation. J Biol Chem. 2014;289(24):17111-23.

9. Stanton WR, Janda M, Baade PD, Anderson P. Primary prevention of skin cancer: a review of sun protection in Australia and internationally. Health Promot Int. 2004;19(3):369-78.

10. Agência Portuguesa do Ambiente. O clima em Portugal: como foi, como é e como será [homepage]. Lisboa: APA; [s.d.]. Available from: http://apambiente.pt/index.php?ref=16\&subref $=81 \&$ sub2 $r e f=118 \&$ su b3ref $=393$

11. Ribeiro C, Relvas A, Carvalho L, Costa V, Gomes L, Costa M. Proteção solar: conhecimentos e hábitos na população pediátrica [Sun protection: knowledge and habits in pediatric population]. Nascer Crescer. 2017;26(1):31-5. Portuguese

12. Gilaberte-Calzada Y, Aguareles-Piracés MJ, Coscojuela-Santaliestra C, Doste-Larrull D, Fajó-Rivas J, Pardos-Martínez C, et al. Factores asociados a la fotoprotección de los niños: una encuesta realizada a los padres [Predictors of sun protection in children: a parental survey]. Actas Dermosifiliogr. 2001;92(3):81-7. Spanish

13. Detert H, Hedlund S, Anderson CD, Rodvall Y, Whiteman DC, Falk M. Validation of Sun Exposure and Protection Index (SEPI) for estimation of sun habits. Cancer Epidemiol. 2015;39(6):986-93.

14. Castilho IG, Sousa MA, Leite RM. Photoexposure and risk factors for skin cancer: an evaluation of behaviors and knowledge among university students. An Bras Dermatol. 2010;85(2):173-8.

15. Bonfá R, Martins-Costa GM, Lovato B, Rezende R, Belletini C, Weber MB. Evaluation of the knowledge and photoprotection habits of children and their caregivers in the city of Porto Alebre, Brazil. Surg Cosmet Dermatol. 2014;6(2):148-53.

16. Haack RL, Horta BL, Cesar JA. Queimadura solar em jovens: estudo de base populacional no sul do Brasil [Sunburn in young people: population-based study in Southern Brazil]. Rev Saude Publica. 2008;42(1):2633. Portuguese

17. Westerdahl J, Ingvar C, Mâsback A, Olsson H. Sunscreen use and malignant melanoma. Int J Cancer. 2000;87(1):145-50.

18. Silva LR, França-Botelho AC. Proteção solar para crianças: estudo preliminar sobre conhecimentos e atitudes dos pais [Sun protection for children: preliminary study on the knowledge and attitudes of parents]. Ciência Saúde. 2011;4(1):2-6. Portuguese

19. Tresoldi GM, Duarte MF, Costa MC. Ensaio comparativo: diferentes sexos relacionando a preocupação com o fotoenvelhecimento [Comparative essay: different sexes relating the concern with photoaging]. Rev Terra \& Cultura: Cad Ensino Pesq. 2019;34(special):164-70. Portuguese 
20. Araujo-Soares V, Rodrigues A, Presseau J, Sniehotta FF.Adolescent sunscreen use in springtime: a prospective predictive study informed by a belief elicitation investigation. J Behav Med. 2013;36(2):109-23.

21. Vieth R. Vitamin D supplementation 25-hydroxyvitamin D concentrations, and safety. Am J Clin Nutr. 1999;69(5):842-56.

22. Malabanan A, Veronikis IE, Holick MF. Redefining vitamin D insufficiency. Lancet. 1998;351(9105):805-6.

23. Maia M, Maeda SS, Marcon C. Correlação entre fotoproteção e concentrações de 25 hidroxi-vitamina D e paratormônio [Correlation between photoprotection and 25 hydroxyvitamin $D$ and parathyroid hormone levels]. An Bras Dermatol. 2007;82(3):233-7. Portuguese

24. Lowe JB, McDermott LJ, Stanton WR, Clavarino A, Balanda KP, McWhirter $B$. Behavior of caregivers to protect their infants from exposure to the sun in Queensland Australia. Health Educ Res. 2002;17(4):405-14.

25. Marques DR, Duarte MC. A radiação solar e protectores solares: conhecimentos e práticas de alunos do $9^{\circ}$ e $11^{\circ}$ anos de escolaridade [Sunscreen and its working mechanism: a study about students' understanding and practices]. Rev Elect Enseñanza Ciencias. 2008;7(3):722-45. Spanish

26. Parrott R, Lemieux R. When the worlds of work and wellness collide: the role of familial support on skin cancer control. J Fam Commun. 2003;3(2):95-106
27. Silva KW, Silva SK, Gomes DC, Santos IM, Dabbur FS. Fotoexposição solar e fotoprotecão em usuários dos serviços de saúde de um centro universitário em Maceió/AL [Internet]. In: $2^{\circ}$ Congresso Nacional de Enfermagem (CONENF), 7-11 de maio de 2018. Available from: https://eventos.set.edu.br/index.php/conenf/article/view/9423/4277

\section{CONFLITO DE INTERESSES}

Os autores declaram não possuir quaisquer conflitos de interesse.

\section{FINANCIAMENTO DO ESTUDO}

Os autores não receberam qualquer financiamento para o desenvolvimento do estudo.

\section{ENDEREÇO PARA CORRESPONDÊNCIA \\ Cátia Alexandra Granja Ferreira \\ E-mail: catigranja@gmail.com \\ https://orcid.org/0000-0001-7759-5135}

Recebido em 04-03-2020

Aceite para publicação em 15-06-2020

\section{ABSTRACT}

\section{EVALUATION OF THE KNOWLEDGE AND PHOTOPROTECTION HABITS OF CHILDREN AND ADOLESCENTS}

Aim: To evaluate the characteristics of the knowledge and sun protection habits of children.

Study: Descriptive and analytic cross-sectional study.

Place: In outpatient, our pediatric emergency department, hospital-level II, and in the child health consultation of a local USF. Population: Children under the age of 18 years and their caregivers who attended the units mentioned.

Methods: Application of a questionnaire on knowledge and habits concerning sun protection, voluntary and confidential answer. Statistical data were processed using the Statistical Package for the Social Sciences - SPSS ${ }^{\circledR}$ program. Statistical significance was considered for $p$-values $<0.05$.

Results: Sample totaled 295, 63.7\% caregivers of children under nine years and $36.3 \%$ adolescents between 10 to 17 years. The period of the day of greatest exposure to sunlight was before 11:00 am and after 4:00 pm (77\%). They use sunscreen, 93\% of adolescents and $90.6 \%$ of children, however, the majority of the population only use it on the beach $(61 \%)(p<0.05)$. As for the frequency of renewal of sunscreen, $40.2 \%$ of the adolescents renewed two times and $52.6 \%$ of the children only once $(p<0.05)$. It is the female who responds to use more umbrella $(76.2 \%)(p<0.05)$ and more girls use sun protection factor (SPF) $30(59 \%)$ and $50+(73.7 \%)$ while more boys use SPF $<15(76.9 \%)(p<0.05)$. Most of those included in the study showed to acquire more information/knowledge about sun protection through the media (46.8\%).

Conclusion: The present study's data demonstrated inadequate sun protection habits, results in line with other studies. It warns of the need for wider dissemination of adequate knowledge that consolidates healthy practices in relation to sun exposure. The multidisciplinarity between primary health care and Pediatrician becomes crucial.

Keywords: Child; Knowledge; Habits; Sunlight; Sunscreening agents. 